\title{
Dependence of Contact Resistance on Current for Ohmic Contacts to Quantized Hall Resistors
}

\author{
Kevin C. Lee
}

\begin{abstract}
The dependence of contact resistance on current has been measured for a large number of ohmic contacts to quantized Hall resistors under quantum Hall effect conditions. Five different functional forms of current dependence are observed at low currents. The trend from best to worst quality can be correlated with the density of defects in the contact, regardless of the physical cause of the defects. The consequences of different types of contact resistance current dependence on the metrological use of samples are discussed.
\end{abstract}

Index Terms - Breakdown, contact degradation, contact resistance, corrosion, current dependence, electrically active defects, ohmic contacts, quantized Hall resistor, quantum Hall effect.

\section{INTRODUCTION}

$\mathbf{E}$ XCESSIVE resistance in the contacts on quantized Hall resistors (QHR's) can cause an increased load on the current source, noisy Hall voltages, and deviations of the measured Hall voltage from its ideal value [1]-[3] making the device unsuitable for use as a resistance standard. It is important to measure all contact resistances over a range of current from 0 to $\pm 100 \mu \mathrm{A}$, for while the contact resistances at the measurement current must clearly be known, high contact resistances at lower currents have a significant effect on measurements, particularly when multiple series connections are used [4].

In this work, the resistances of potential probe contacts on over 15 QHR's with alloyed ohmic contacts prepared in different manners were measured. These contacts varied in quality from excellent to quite poor. The physical causes for the poor contact quality varied from physical damage to the contact caused by excessive wire bonding pressure, to corrosion, to poor morphology of the metal-semiconductor interface produced during alloying. The current dependencies of the contact resistances in all cases, however, appear to follow a common trend. This paper describes this trend, shows that it is consistent with the effects of increasing disorder in the contact, and describes the sometimes significant effect of current dependent contact resistances on the use of QHR's as resistance standards.

\section{EXPERIMENTAL PROCEDURE}

Eleven of the QHR's tested in this work were prepared with $\mathrm{AuGe} / \mathrm{Ni}$ contacts from $\mathrm{GaAs} / \mathrm{Al}_{x} \mathrm{Ga}_{1-x} \mathrm{As}$ heterostructures grown using metal organic chemical vapor deposition

Manuscript received July 2, 1998.

The author is with the National Institute of Standards and Technology, Gaithersburg, MD 20899 USA (e-mail: kevin.lee@nist.gov).

Publisher Item Identifier S 0018-9456(99)03221-0.
(MOCVD) by the Laboratoires d' Electronique Philips (LEP) ${ }^{1}$ under contract to the EUROMET consortium [5], [9]. In addition, several samples were prepared at the National Institute of Standards and Technology (NIST) by alloying indium to GaAs/ $/ \mathrm{Al}_{x} \mathrm{Ga}_{1-x} \mathrm{As}$ heterostructures grown using molecular beam epitaxy (MBE).

The devices were cooled in a cryostat to temperatures between 1.1 and $1.4 \mathrm{~K}$ in magnetic flux densities between 4 and $8 T$, as required to observe the $i=4(6453.201750 \Omega)$ quantum Hall resistance plateau. Contact resistances were measured using a technique similar to that described in [6] and described in detail in [9]; the magnetic flux density was set to a value at the center of the $i=4$ plateau and current was passed between a contact "A" and a second contact, usually the source or drain at the end of the Hall bar (see inset to Fig. 1). The potential was measured between contact " $A$ " and a second contact "B" that did not carry current and which was at nominally the same potential as contact "A." The current was increased by an increment $\delta I$, between 0.5 and $10 \mu \mathrm{A}$, and the voltage was measured again. The "differential contact resistance" was determined by dividing the difference in the two voltages by the current increment. This resistance includes the contact resistance of the contact and the resistance of the wire in the cryostat probe, which varies between 1.2 and 1.6 $\Omega$. In the earliest experiments, the current was initially set to $-I_{\max }$ and increased in steps to the positive limit $+I_{\max }$; in later experiments, the current was increased in steps from zero to a positive limit $\mathrm{I}_{\max }$, usually $100 \mu \mathrm{A}$, then reset to zero, and decreased to $-I_{\max }$. The contact resistance measurements were made over a fairly short period of time (between 1 and $3 \mathrm{~min}$ ). The standard uncertainty in the measurement of resistance was about $0.3 \Omega$; the uncertainty in the measurement of current was less than $0.03 \mu \mathrm{A}$.

\section{RESULTS}

The variation of the resistance of a contact with current arises from the action of two different factors, which operate over different ranges of current. The influence of the first factor is observed at lower currents, and that of the other, independent factor, at higher currents. The exact ranges of current over which the two types of behavior are observed is a function of the dimensions of the sample. For the potential probes

\footnotetext{
${ }^{1}$ Certain commercial equipment, instruments, or materials are identified in this paper to foster understanding. Such identification does not imply recommendation or endorsement by the National Institute of Standards and Technology, nor does it imply that the materials or equipment identified are necessarily the best available for the purpose.
} 


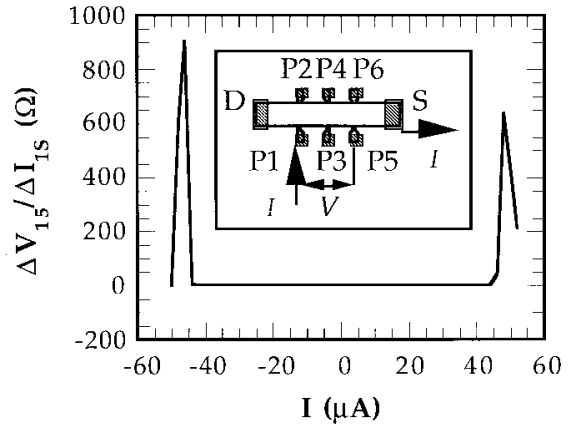

Fig. 1. Contact resistance versus current for ideal contact. Data from LEP sample with $\mathrm{Si}_{3} \mathrm{~N}_{4}$ coating, serial \#E7C. Inset shows probe numbering.

on the LEP samples, "low-current behavior" is generally observed when the magnitude of the current is less than about $50 \mu \mathrm{A}$, and "high current behavior" is observed when the magnitude of the current is greater than about $80 \mu \mathrm{A}$. Five different functional forms of "low current behavior" have been observed and are described in the first part of this section; two different functional forms of "high current behavior" have been observed and are described in the second part of this section.

\section{A. Low-Current Behavior}

The dependence of contact resistance on current for low currents for all of the samples tested in this work fell into one of five different categories.

1) Ideal Contact: The contact resistance vanishes for currents with magnitude less than a critical current $I_{c}$, which on the best samples is between 35 and $55 \mu \mathrm{A}$ for the potential probes on the LEP samples. $I_{c}$ is proportional to the width of the narrowest part of the heterostructure through which current must pass. At currents with magnitude above $I_{c}$, the contact resistance abruptly increases (see Fig. 1), probably due to breakdown of the quantum Hall effect [7], usually to several hundred ohms and seldom more than $1 \mathrm{k} \Omega$.

2) Nearly Ideal Contact: Contacts that are of slightly worse quality exhibit the same general form of current dependence as an ideal contact; the contact resistance vanishes for currents $|I| \leq I_{c}$, but $I_{c}$ is smaller, varying between 0 and $30 \mu \mathrm{A}$ for potential probes on the LEP samples.

3) Limiting Case of Ideal Contact: In the limiting case, the contact resistance only vanishes at zero current, as shown in Fig. 2.

4) Poor Quality Contact: Contacts with worse quality exhibit a current dependence similar to the limiting case of an ideal contact, except that the contact resistance does not vanish at zero current. While the value of the contact resistance and the range over which it varies can differ widely from contact to contact, in all cases, the form of the current dependence is essentially the same.

5) Inverted Current Dependence: Contacts with the worst quality have resistances that actually reach a maximum when the lowest currents are passed through the device, as shown in Fig. 3. The values of the contact resistances of the contacts that exhibit this form of current dependence can vary over a wide range. The maximum contact resistance can be as little

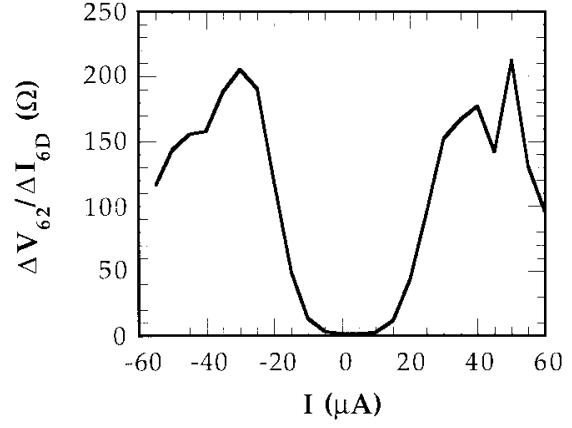

Fig. 2. Contact resistance versus current for limiting case of ideal contact. Data from LEP sample without $\mathrm{Si}_{3} \mathrm{~N}_{4}$ coating, serial \#E6. The magnetic flux density was set to a value that minimized $V_{26}$.

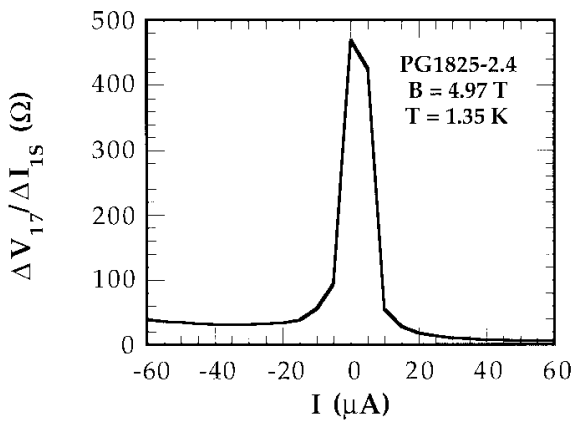

Fig. 3. Contact resistance versus current for contact with inverted current dependence. Data from sample PG1825-2.4, with alloyed indium contacts. N.B.: This sample had ten potential probe contacts.

as 14 to $20 \Omega$, and the minimum contact resistance measured at currents approaching $100 \mu \mathrm{A}$ can be negligibly small, while in other cases, contact resistances can be as high as thousands of ohms throughout the range $\pm 100 \mu \mathrm{A}$.

\section{B. High-Current Behavior}

The contact resistance current dependence of all contacts tested at higher currents fell into one of two different categories.

1) OHMIC Contact: The majority of contacts (all but about a dozen) exhibited at high currents contact resistances that were more or less independent of current and were the same for positive and negative current directions, as observed by [8]. The contacts from which the data in Figs. 1-3 were obtained had ohmic contact resistances in this high current regime.

2) Rectifying Contact: About a dozen contacts were observed to exhibit rectifying characteristics, with resistances increasing sharply at negative currents with magnitudes greater than 50 to $80 \mu \mathrm{A}$ while remaining low for positive currents of the same magnitude, as shown in Fig. 4.

Interestingly, the behavior of the contact resistance at high currents with magnitude above 50 to $80 \mu \mathrm{A}$ (ohmic or rectifying) was independent of the behavior of the contact at lower currents with magnitude less than 30 to $50 \mu \mathrm{A}$. An example of a rectifying contact exhibiting nearly ideal current dependence at low currents is shown in Fig. 4, but other rectifying contacts were found that exhibited at low currents nearly all of the other types of current dependence described in the first part of this section. 


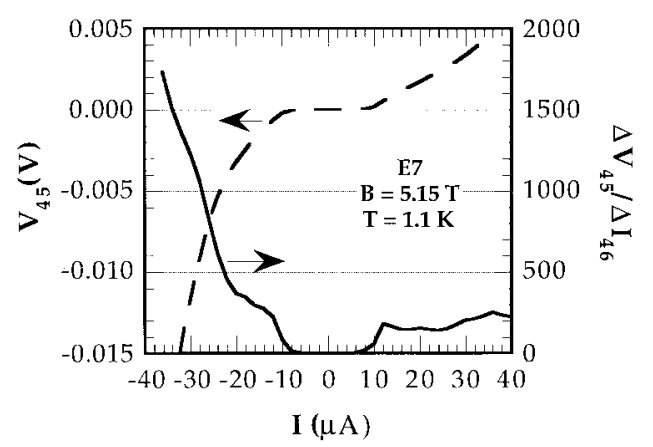

Fig. 4. Contact resistance (solid curve) and voltage across contact (dashed curve) versus current for rectifying contact. Data from uncoated LEP sample E7.

\section{DISCUSSION}

\section{A. Correlation of Current Dependence with Defect Density}

The current dependencies described above have been observed with contacts prepared using different techniques as well as contacts that have been subjected to treatments known to cause electrically active defects in the contact region. This evidence indicates that the trend in contact resistance current dependence from ideal to inverted current dependence described above is correlated with an increasing density of electrically active defects in the contact region, shown as follows.

1) The contacts on the LEP samples, both with and without silicon nitride coatings, were all ideal or nearly ideal when the samples were delivered to NIST in 1990. The best alloyed indium contacts prepared on QHR devices fabricated at NIST also exhibit ideal characteristics, indicating that this type of current dependence is typical of high quality contacts with a low density of electrically active defects.

2) The resistances of contacts on the LEP samples coated with a protective silicon nitride layer are still all ideal or nearly ideal. As discussed in [9], the silicon nitride layer prevented corrosive compounds in the atmosphere from altering the chemical structure of the contact, so these contacts have essentially the same structure as they did when the samples were made in 1990.

3) The contacts on the LEP samples that were not coated with silicon nitride (and hence were exposed to corrosive compounds in the atmosphere) are now no longer ideal. Alloyed AuGe/Ni contacts of the type made to the LEP samples will suffer changes in chemical structure, with the creation of electrically active defects in the contact region, when exposed to normal atmospheric conditions for a period of many years [9]. The changes in the current dependence of the resistance of these contacts can be attributed to an increase in density of electrically active defects with time as the contacts have corroded.

4) Contacts on LEP samples (sample E6C) to which 25 $\mu \mathrm{m}$ diameter gold wires were bonded directly over the heterostructure show inverted current dependence. It is known that the stresses on the heterostructure created during bonding will create electrically active defects in the contact region [6]. This is further evidence that inverted current dependence can be attributed to electrically active defects in the contact region, in this case caused by mechanical damage. The fact that aged uncoated LEP samples also exhibit similar current dependence indicates that the electrically active defects caused by corrosion-induced chemical changes in the structure of the contact and mechanical damage both have similar effects on the contact's properties, giving rise to nonideal behavior.

5) Alloyed indium contacts that have smooth, uniform interfaces between the metallic indium and the heterostructure exhibit ideal behavior. Contacts with poor quality, nonuniform interfaces exhibit behaviors ranging from nearly ideal to rectifying, again indicating that the nonideal behaviors can be attributed to electrically active defects at the interface between the contact metal and the two-dimensional electron gas (2 DEG).

These observations indicate that the trend in contact resistance current dependence from ideal to rectifying can be correlated with an increasing density of electrically active defects in the region between the metallic contact and the 2 DEG. Furthermore, the defects caused by mechanical damage and chemical corrosion have a very similar effect on the current dependence of the contact resistance.

The exact mechanism by which these defects cause the different types of current dependence at lower currents is not clear, but it appears that this behavior is strongly influenced by quantum mechanical effects related to the quantization of the conduction electrons into Landau levels, and the observations described above are consistent with at least two published theories. van Son et al. [10] have proposed that disorder in the contact region gives rise to a nonequilibrium distribution of electrons in the Landau levels, which in turn causes breakdown of the dissipationless transport characteristic of the quantum Hall effect (QHE). Increasing densities of defects will result in increased scattering of electrons, with a consequent decrease in the critical current, and an increase in the contact resistances, as observed in this work.

Cage et al. [11] have proposed that when the current through an ideal contact is increased, the Hall voltage increases, and eventually becomes high enough to cause the electric field in certain regions of the sample to reach a critical value that permits electrons to tunnel from the highest occupied Landau level to the lowest unoccupied Landau level, a process called quasi-elastic inter-Landau level scattering. Under these conditions, breakdown of the QHE occurs, and the contact resistances increase sharply. Increasing densities of electrically charged defects will create regions of the sample in which this critical electric field is reached at lower Hall fields and lower currents, consistent with the observations reported in this paper.

The high current behavior of the contacts (ohmic and rectifying) is not well understood, but it appears that the conduction at high currents is not significantly influenced by the quantization of electrons into Landau levels. The rectifying current dependence observed in some contacts at higher 
currents may possibly be due to the presence of a potential barrier between the metal contact and the semiconductor.

\section{B. Effect of Current Dependent Contacts on Use of QHR's as Resistance Standards}

In order to evaluate the effect of contact resistance on the use of a QHR as a resistance standard, it is essential that the contact resistance be measured at a current close to the value that will be passed through it during precision measurements or calibrations. For example, if one passes a current of $50 \mu \mathrm{A}$ through the source and drain contacts when making precision measurements, it is tempting to use this current source to test the contact resistances of both the current and potential probes prior to making precision measurements. While this would be appropriate for the current contacts, it could give misleading results for the potential contacts. As can be seen from Fig. 1, even if the potential contact is ideal, the critical current for potential contacts on the LEP devices can be as low as $50 \mu \mathrm{A}$. Measurement of the resistance of such a contact at $50 \mu \mathrm{A}$ could give a value as high as several hundred ohms. Since little or no current is drawn through the potential probes during precision measurement and since the contact shown in Fig. 1 has vanishingly small resistance at low currents, the device would be usable as a resistance standard, in apparent contradiction to the specifications in [1] that the contact resistance be less than $10^{-4}\left(R_{K} / i+R_{L}\right)$ where $R_{K}$ is von Klitzing's constant, defined to be $25812.807 \Omega, i$ is an integer identifying the quantized Hall resistance plateau, and $R_{L}$ is the lead resistance. In practice, the guidelines are not violated, for if the potential probe's contact resistance had been measured with a current less than the critical current, it would have met the specification in [1]. Several authors have reported being able to use devices with high contact resistances as resistance standards [1], [5], in apparent contradiction to the specifications in [1], but none of these authors have reported the currents used to measure the contact resistances, the critical currents of the potential probe contacts, or the dependence of the contact resistances on current. While it is possible that the devices tested by these authors actually had ideal or nearly ideal contacts, but the authors measured the contact resistances only at selected currents that were close to or slightly higher than the critical current for an ideal potential contact on these devices, this cannot be determined from the published data. Because non-negligible contact resistances can have serious effects on precision measurements of the quantized Hall resistance [1], it is essential that if contact resistances are measured at a single current, that current be close to the one that will flow through the device during precision measurements: for current contacts, the current should be the measurement current, and for potential probe contacts, the current should be quite small, e.g., less than $1 \mu \mathrm{A}$.

As a second example, measurement of the current dependence of potential probe contact resistances is particularly important when multiple series connections of a QHR are used, as is commonly done in ac measurements of the quantized Hall resistance. When a double series connection of a QHR is used [4, Sections 6.1 and 7.1] currents as high as $16 \mathrm{nA}$ can flow through the potential probe contact. In the worst case, a contact with inverted current dependence (as shown in Fig. 4) could appear to have a resistance of less than a few ohms if measured with a current equal to the measurement current $(39.9 \mu \mathrm{A}$ in [4]). One would then conclude that the relative difference between the measured $R_{X Y}$ and $R_{H}=R_{K} / i$, would be of the order of $\left[\left(R_{X Y}-R_{H}\right) / R_{H}\right]=3 \times 10^{-7}$ for the $i=2$ plateau (for the conditions used in [4]). In fact, the resistance of this contact would be nearly $500 \Omega$ at the current of $16 \mathrm{nA}$ actually flowing through it when the QHR measurement was made. The correct relative difference between $R_{X Y}$ and $R_{H}$ would be $1.5 \times 10^{-5}$, over an order of magnitude larger.

These two examples demonstrate that contact resistances measured at a single current different from the current that will flow through the contact during precision measurements cannot be used to judge whether the device will be of standards quality. The contact resistance of each contact must be measured at the current that will be passed through it during measurement. The current-contact (source and drain) resistances must at least be measured at the measurement current (with current flowing in both directions), and the potential probe contact resistances must be measured over a range of small currents about $I=0$. If multiple series connections are used, the contact resistance of the potential probe must be measured at the current that will pass through it during QHE measurements.

\section{CONCLUSION}

The current dependence of the resistances of ohmic contacts to QHR devices subjected to damage-inducing treatments including corrosion and mechanical damage exhibits a definite trend that can be correlated with different densities of electrically active defects in the region between the metallic contact and the heterostructure. This correlation is consistent with several theories relating contact disorder to the breakdown of the QHE. The form of the current dependence is very important for evaluating whether a sample is of standards quality. Because contact resistances can vary over a range of several orders of magnitude with relatively small changes in current, measurements of contact resistance at a single current not equal to the current that will be passed through the contact during QHE measurements can result in very large errors.

\section{ACKNOWLEDGMENT}

The author would like to thank C. Lee, Cornell University, M. Cage and A. Clark, NIST, for many helpful discussions, and the Calibration and Coordination Group of the Department of Defense for supporting this work.

\section{REFERENCES}

[1] F. Delahaye, "Technical guidelines for reliable measurements of the quantized Hall resistance," Metrologia, vol. 26, pp. 63-68, 1989.

[2] W. van der Wel, E. G. Haanappel, J. E. Mooij, C. J. P. M. Harmans, J. P. André, G. Weimann, K. Ploog, C. T. Foxon, and J. J. Harris, "Selection criteria for AlGaAs-GaAs heterostructures in view of their use as a quantum Hall resistance standard," J. Appl. Phys., vol. 65, pp. 3487-97, May 1989. 
[3] B. Jeckelmann and B. Jeanneret, "Influence of the voltage contacts on the four-terminal quantized Hall resistance in the nonlinear regime," IEEE Trans. Instrum. Meas., vol. 46, pp. 276-280, Apr. 1997.

[4] A. M. Jeffery, R. E. Elmquist, and M. E. Cage, "Precision tests of a quantum Hall effect device DC equivalent circuit using double series and triple series connections," J. Res. Nat. Inst. Stand. Technol., vol. 100, pp. 264-268, Apr. 1993.

[5] F. Piquemal, G. Genevés, F. Delahaye, J. P. André, J. N. Patillon, and P. Frijlink, "Report on a joint BIPM-EUROMET project for the fabrication of QHE samples by the LEP," IEEE Trans. Instrum. Meas., vol. 42, pp. 264-268, Apr. 1993.

[6] K. C. Lee, "Bonding wires to quantized Hall resistors," IEEE Trans. Instrum. Meas., vol. 44, pp. 249-252, Apr. 1995.

[7] M. E. Cage, R. F. Dziuba, B. F. Field, E. R. Williams, S. M. Girvin, A. C. Gossard, D. C. Tsui, and R. J. Wagner, "Dissipation and dynamic nonlinear behavior in the quantum Hall regime," Phys. Rev. Lett., vol 51, pp. 1374-1377, 1983.

[8] H. Bruus, "The quantum Hall effect in $\mathrm{GaAs} / \mathrm{Ga}_{1-x} \mathrm{Al}_{x}$ As heterostructures," Ph.D. dissertation, H. C. Ørsted Institute, Univ. Copenhagen, Denmark, 1990, pp. 119-130.

[9] K. C. Lee, "Degradation of GaAs/AlGaAs quantized Hall resistors with alloyed AuGe/Ni contacts," J. Res. Nat. Inst. Stand. Technol., vol. 103 , pp. 177-200, Mar. 1998.
[10] P. C. van Son, G. H. Kruithof, and T. M. Klapwijk, "Current contacts and the breakdown of the quantum Hall effect," Phys. Rev., vol. B42, pp. 11267-11275, Dec. 1990.

[11] M. E. Cage and C. F. Lavine, "Using quantized breakdown voltage signals to determine the maximum electric fields in a quantum Hall effect sample," J. Res. Nat. Inst. Stand. Technol., vol. 100, pp. 269-276, May 1995.

Kevin C. Lee received the B.S., M.S., and Ph.D. degrees in applied physics from Cornell University, Ithaca, NY. His dissertation concerned the fabrication and characterization of electronic devices on submicrometer-thick, freestanding, single-crystal membranes of semiconductors.

Since 1988, he has been with the Fundamental Electrical Measurements Group, National Institute of Standards and Technology (NIST), Gaithersburg, $\mathrm{MD}$, where he is developing techniques to make quantized Hall resistors, and designing and testing new measurement systems for use with quantum Hall resistors. 